\title{
Cognitive Behavioral Therapy for Insomnia Reduces Hypnotic Prescriptions
}

\author{
Kyung Mee Park', Tae Ho Kim¹, Woo Jung Kim², Suk Kyoon An¹, Kee Namkoong', and Eun Lee ${ }^{1 凶}$ \\ ${ }^{1}$ Institute of Behavioral Science in Medicine and Department of Psychiatry, Yonsei University College of Medicine, Seoul, Republic of Korea \\ ${ }^{2}$ Department of Psychiatry, Seonam University College of Medicine, Myongji Hospital, Goyang, Republic of Korea
}

Objective This study determined whether cognitive behavioral therapy for insomnia (CBT-i) decreased the need for sleep medications and produced better treatment outcomes than pharmacotherapy alone.

Methods We reviewed data from patients with insomnia in the outpatient clinic of a general hospital between 2009 and 2015 . We compared 41 patients who received five sessions of CBT-i with 100 age- and sex-matched patients who received pharmacotherapy only. We evaluated the change in prescription for sleep (i.e., antidepressants, hypnotics, and others) between the first and last visits using repeated measures analysis of variance (ANOVA). Clinical global impressions and completion status at the last visit were assessed using the chisquare test.

Results We found a significant decrease in the prescription rate and the dosage of hypnotics among patients who received CBT-i when compared with control patients. There was no significant change in the dosage of antidepressants between the two groups. Achievement of case closure was better in the CBT-i group at the trend level. Clinical global impression at the last visit was not significantly different.

Conclusion These results show that CBT-i reduces the need for hypnotics among insomnia patients. Our results indicate that CBT-i offers additional benefits beyond improving sleep characteristics and thus provides another reason for recommending CBT-i as a first-line treatment for insomnia.

Psychiatry Investig 2018;15(5):499-504

Key Words Insomnia, Hypnotics, Cognitive behavioral therapy, Cognitive behavioral therapy for insomnia, Sleep.

\section{INTRODUCTION}

The Diagnostic and Statistical Manual of Mental Disorders defines insomnia as dissatisfaction with sleep quantity or quality accompanied by difficulty in initiating sleep, difficulty in maintaining sleep, or early-morning awakening with an inability to return to sleep despite adequate opportunity for sleep. If these sleep disturbances cause clinically significant distress in important areas of functioning and occur at least three nights per week for at least three months, a diagnosis of insomnia is warranted. ${ }^{1}$ Insomnia is receiving increasing attention because of its impact on quality of life, relationship to other medical conditions, ${ }^{2,3}$ and psychiatric disorders, ${ }^{4,5}$ and

Received: August 1, 2017 Revised: October 10, 2017

Accepted: November 20, 2017

$\triangle$ Correspondence: Eun Lee, MD, PhD

Department of Psychiatry, Yonsei University College of Medicine, 50-1 Yonseiro, Seodaemun-gu, Seoul 03722, Republic of Korea

Tel: +82-2-2228-1620, Fax: +82-2-313-0891, E-mail: leeeun@yuhs.ac

(c) This is an Open Access article distributed under the terms of the Creative Commons Attribution Non-Commercial License (http://creativecommons.org/licenses/bync/4.0) which permits unrestricted non-commercial use, distribution, and reproduction in any medium, provided the original work is properly cited. association with increased mortality rates among patients with congestive heart failure, coronary diseases, and metabolic diseases. ${ }^{6}$ Insomnia is quite common, affecting $8-17 \%$ of the general population, ${ }^{7,8}$ and its prevalence increases with age, lower socioeconomic status, and concurrent medical and psychiatric comorbidity. ${ }^{9}$ However, only $5 \%$ of individuals with chronic insomnia seek help for their sleep problems from a health care provider. ${ }^{10}$

Guidelines recommend cognitive behavioral therapy for insomnia (CBT-i) as a first-line treatment for insomnia. ${ }^{11,12}$ Despite these guidelines, doctors still routinely prescribe hypnotics for insomnia. ${ }^{13}$ Although hypnotics effectively reduce the symptoms of insomnia, they cause many side effects including increased risk of falls resulting in fracture, cognitive impairment, impaired motor coordination, sedation, confusion, motor vehicle accidents, and dependence. ${ }^{14,15}$ In contrast, CBT-i treats insomnia by modifying thought patterns and behaviors that reinforce poor sleep, with minimal side effects and no potential for dependency. Previous studies show that CBT-i improves sleep efficiency, increases total sleep time, and reduces wake time after sleep onset in patients with in- 
somnia. ${ }^{16-18} \mathrm{CBT}-\mathrm{i}$ exerts similar short-term therapeutic effects, but better long-term therapeutic effects on insomnia symptoms than hypnotics. ${ }^{19}$ However, there are some barriers to administering CBT-i to insomnia patients. Changing thought patterns and behaviors takes more effort than taking medication, and patience is required to see treatment effects. Furthermore, patients may not want to visit the clinic every week to receive CBT-i. For these reasons, patients and clinicians are reluctant to use CBT-i despite knowledge of its benefits, and insomnia patients often receive pharmacotherapy in addition to CBT-i.

This study determined whether insomnia patients who received CBT-i had 1) a reduced need for sleep medications and 2) better treatment outcomes during long-term followup than control patients who received pharmacotherapy only. Using a retrospective study design, we reviewed outpatient clinic data from a psychiatry department in a general hospital setting and compared prescribed medication dosages and treatment outcomes between CBT-i and control groups.

\section{METHODS}

\section{Participants}

We reviewed electronic medical records of patients diagnosed with and initiating treatment for insomnia (G47.0) or primary insomnia (F51.0) in the Severance Hospital outpatient clinic from January 2009 to December 2014. We found $41 \mathrm{pa}-$ tients who completed five sessions of CBT-i as confirmed by doctors' orders and payment statements. For comparison, we selected 100 outpatients with the same diagnoses who did not receive CBT-i during the same period matched for age, sex, and medical comorbidity. We excluded those cases diagnosed with major psychiatric disorders including schizophrenia spectrum and other psychotic disorders, bipolar I disorder, major depressive disorder, persistent depressive disorder, substance use disorder, or sleep apnea as a principal diagnosis in order to focus on insomnia treatment. Because of the small sample size, we did not exclude any psychiatric diagnosis for the CBT-i group. As a result, we included 141 patients. We followed up using their medical records through December 2015 for the evaluation of successful treatment. The Institutional Review Board of Severance Hospital approved this study (4-2015-1027).

\section{Procedures}

Our CBT-i program was based on Edinger's CBT-i proto$\mathrm{col}^{20}$ The program was implemented as five weekly 90 -minute therapy sessions discussing an introduction to the method, stimulus control, sleep restriction, sleep hygiene, and cognitive therapy and trouble-shooting. We provided the treatment program to groups of 2-4 persons served by two mental health professionals (one psychiatrist and one nurse). As the CBT-i program is not an exclusive treatment arm in our hospital, patients could also receive pharmacotherapy. In this data, CBTi was performed between January 2009 and December 2014.

\section{Measurement}

We reviewed the electronic medical records to obtain information about patients' sex, age, comorbid medical and psychiatric conditions, prescribed medications for sleep, total number of clinic visits, first and last visit dates, total duration of follow-up, clinical global impression (CGI), and status of case closure at the last visit as indicated by doctors' comments. Because most patients undergoing CBT-i also were prescribed medications for sleep, we evaluated the additive effect of CBT$i$ to their treatment regimen. As the benefits of CBT-i are evident after 12 months of treatment, ${ }^{19,21}$ we investigated 1) changes in the need for sleep medications between the first and last visits and 2) treatment outcome at the last visit.

To evaluate changes in the need for sleep medications, we reviewed data on all prescribed medications for sleep at the first and last clinic visits. We sorted prescribed medications into three categories: hypnotics, antidepressants, and other medications. In the case of hypnotics, we separated them into two categories based on elimination half-lives: shortacting ( $<24$ hours) and long-acting ( $\geq 24$ hours) hypnotics. ${ }^{22}$ We investigated the prescription rate and the dosage of sleep medication at the first and the last visit. We also examined the prescription rate and dosage of mirtazapine and trazodone specifically because of their frequent off-label use for insomnia. ${ }^{23}$ To evaluate the prescribed dosages, we converted the antidepressant dosage to dose equivalent of fluoxetine $e^{24,25}$ and the hypnotic dosage to dose equivalent of lorazepam. ${ }^{26}$

To evaluate treatment outcome, we assessed doctors' comments at the last visit and categorized CGI as follows: improvement, no change, or decline. We also assessed the status of case closures, which were defined as meeting two conditions: 1) doctor's comment that specified case closure on the last outpatient clinic visit and 2) absence of next visit appointment. If there was no comment about case closure, we defined it as "lost to follow-up." If a next visit appointment was present after $12 / 31 / 2015$, the case was defined as "treatment maintenance."

\section{Statistical analysis}

We performed Chi-square statistical analysis to compare prescription rates from the first to the last visits and between the CBT-i and the control group. We also conducted repeated measures analysis of variance (ANOVA) to examine the change in prescribed dosage of hypnotics and antidepressants from 
the first to last visits between the two groups. We did not analyze the converted dosage of other medications because of their relatively low occurrence $(<5 \%)$. We excluded "as needed" prescriptions, because it was difficult to know exact use of these medications. Comparison of CGI and status of case closure between the two groups were performed using chisquared analysis. Independent sample t-tests and chi-squared tests were used to compare continuous and categorical variables between CBT-i and control patients, respectively. Data analyses were conducted using SPSS for Windows version 20 (IBM Corp., Armonk, NY, USA), and statistical significance was set at $\mathrm{p}<0.05$.

\section{RESULTS}

\section{Demographic and clinical characteristics}

Patients ranged in age from 20 to 84 years. Within the CBTi group, psychiatric comorbidities were generalized anxiety disorder $(n=1)$, major depressive disorder $(n=2)$, bipolar II disorder $(n=1)$, acute stress disorder $(n=1)$, and panic disorder $(n=2)$. Within the control group, psychiatric comorbidities were: generalized anxiety disorder $(n=1)$, panic disorder $(n=7)$, somatic symptom disorder $(n=3)$, and social phobia $(n=1)$. We found no significant difference in the frequency of psychiatric comorbidities between groups (Table 1). Among the control patients, five started their psychiatric treatment during admission. None required re-hospitalization for psychiatric comorbidity during the observation period. Their diagnoses were as follows: two adjustment disorders, two depressive disorders, and one anxiety disorder. None were admitted for psychiatric comorbidity in the CBT-i group.

As our institution is a general hospital, approximately onethird of CBT-i and control patients had medical comorbidities, including hypertension, diabetes mellitus, thyroid disease, cardiovascular disease, migraine, herniated cervical discs, and cancer. No patients had uncontrolled medical illness, and there was no significant difference in the frequency of medical comorbidities between groups (Table 1). There were four patients with cancer in the CBT-i group (breast cancer, $\mathrm{n}=2$; thyroid cancer, $\mathrm{n}=1$; acute myeloblastic leukemia, $\mathrm{n}=1$ ) and ten patients with cancer in the control group (advanced gastric cancer, $n=2$; breast cancer, $n=6$; thyroid cancer, $n=1$; sigmoid colon cancer, $n=1$ ). All patients with cancer were in a stable disease state and were not admitted during the study period, except for scheduled chemotherapy (CBT-i group, $\mathrm{n}=1$; control group, $\mathrm{n}=1$ ).

\section{Need for sleep medication}

We compared the differences in the prescription rate between the two groups at their first and the last visit (Table 2).

Table 1. Demographic and clinical characteristics of patients

\begin{tabular}{lccc}
\hline & CBT-i (N=41) & Control (N=100) & $\mathrm{p}$ \\
\hline Mean age, years (SD) & $51.80(14.62)$ & $52.12(14.04)$ & 0.90 \\
Sex, male/female & $10 / 31$ & $26 / 74$ & 0.84 \\
Patients with psychiatric comorbidities, N (\%) & $7(17)$ & $13(13)$ & 0.33 \\
Patients with medical comorbidities, N (\%) & $13^{*}(31)$ & $34^{\dagger}(34)$ & 0.80 \\
Patients with cancer, N (\%) & $4^{\ddagger}(10)$ & $10^{\S}(10)$ & 0.97 \\
Mean duration of total follow-up, days (SD) & $541.85(694.95)$ & $610.09(643.33)$ & 0.58 \\
\hline
\end{tabular}

*hypertension $(\mathrm{N}=3)$, diabetes mellitus $(\mathrm{N}=2)$, chronic heart failure $(\mathrm{N}=1)$, destroyed lung due to old pulmonary tuberculosis $(\mathrm{N}=1)$, cancer $(\mathrm{N}=4)$, thypertension $(\mathrm{N}=8)$, diabetes mellitus $(\mathrm{N}=3)$, coronary artery disease $(\mathrm{N}=3)$, end stage renal disease $(\mathrm{N}=2)$, migraine $(\mathrm{N}=2)$, asthma $(\mathrm{N}=1)$, herniated cervical disc $(\mathrm{N}=1)$, osteoporosis $(\mathrm{N}=1)$, hyperthyroidism $(\mathrm{N}=1)$, aneurysm $(\mathrm{N}=1)$, cancer $(\mathrm{N}=10)$, 拈reast cancer $(\mathrm{N}=2)$, thyroid cancer $(\mathrm{N}=1)$, acute myeloblastic leukemia $(\mathrm{N}=1)$, sbreast cancer $(\mathrm{N}=6)$, advanced gastric cancer $(\mathrm{N}=2)$, thyroid cancer $(\mathrm{N}=1)$, sigmoid colon cancer $(\mathrm{N}=1)$. CBT-i: cognitive behavioral therapy of insomnia, SD: standard deviation

Table 2. Prescribed medications at the first and last visit

\begin{tabular}{|c|c|c|c|c|c|c|}
\hline & \multicolumn{2}{|c|}{ First visit } & \multirow{2}{*}{$\mathrm{p}$} & \multicolumn{2}{|c|}{ Last visit } & \multirow{2}{*}{$\mathrm{p}$} \\
\hline & CBT-i $(\mathrm{N}=41)$ & Control $(\mathrm{N}=100)$ & & CBT-i $(\mathrm{N}=41)$ & Control $(\mathrm{N}=100)$ & \\
\hline Any pharmacotherapy, N (\%) & $35(85)$ & $94(94)$ & 0.11 & $22(53)$ & $91(91)$ & $<0.001$ \\
\hline Hypnotics, N (\%) & $33(81)$ & $92(92)$ & 0.08 & $23(56)$ & $90(90)$ & $<0.001$ \\
\hline Antidepressants, N (\%) & $20(49)$ & $65(65)$ & 0.07 & $12(30)$ & $54(54)$ & 0.01 \\
\hline Others, N (\%) & $2 *(5)$ & $3^{\dagger}(3)$ & 0.63 & $0(0)$ & $5^{\ddagger}(5)$ & 0.32 \\
\hline Short-acting hypnotics, N (\%) & $29(71)$ & $83(83)$ & 0.10 & $20(49)$ & $74(74)$ & 0.004 \\
\hline Long-acting hypnotics, N (\%) & $8(20)$ & $29(29)$ & 0.25 & $6(15)$ & $39(39)$ & 0.005 \\
\hline
\end{tabular}

${ }^{*}$ quetiapine $(\mathrm{N}=2)$, ${ }^{\dagger}$ buspirone $(\mathrm{N}=3),{ }^{\ddagger}$ quetiapine $(\mathrm{N}=2)$ and buspirone $(\mathrm{N}=3)$. CBT-i: cognitive behavioral therapy of insomnia 
Table 3. Comparison of dose changes in prescribed medications for sleep between the CBT-i and control groups

\begin{tabular}{|c|c|c|c|c|c|c|c|c|}
\hline \multirow{2}{*}{ Outpatient visit } & \multirow{2}{*}{ CBT-i } & \multirow{2}{*}{ Control } & \multicolumn{2}{|c|}{ Group } & \multicolumn{2}{|c|}{ Group $\times$ time } & \multicolumn{2}{|c|}{ Time } \\
\hline & & & $\mathrm{F}$ & $\mathrm{p}$ & $\mathrm{F}$ & $\mathrm{p}$ & $\mathrm{F}$ & $\mathrm{p}$ \\
\hline Hypnotics, mean (SD) & & & 7.23 & 0.01 & 14.27 & $<0.001$ & 1.74 & 0.19 \\
\hline First visit & $1.30(1.03)$ & $1.24(0.90)$ & & & & & & \\
\hline Last visit & $0.69(0.85)$ & $1.53(1.14)$ & & & & & & \\
\hline Antidepressants, mean (SD) & & & 0.20 & 0.65 & 1.93 & 0.17 & 0.53 & 0.47 \\
\hline First visit & $3.86(6.04)$ & $3.38(4.20)$ & & & & & & \\
\hline Last visit & $3.44(7.68)$ & $4.70(6.34)$ & & & & & & \\
\hline
\end{tabular}

The antidepressant dosage was converted to dose equivalent of fluoxetine, and the hypnotic dosage was converted to dose equivalent of lorazepam. CBT-i: cognitive behavioral therapy of insomnia, SD: standard deviation

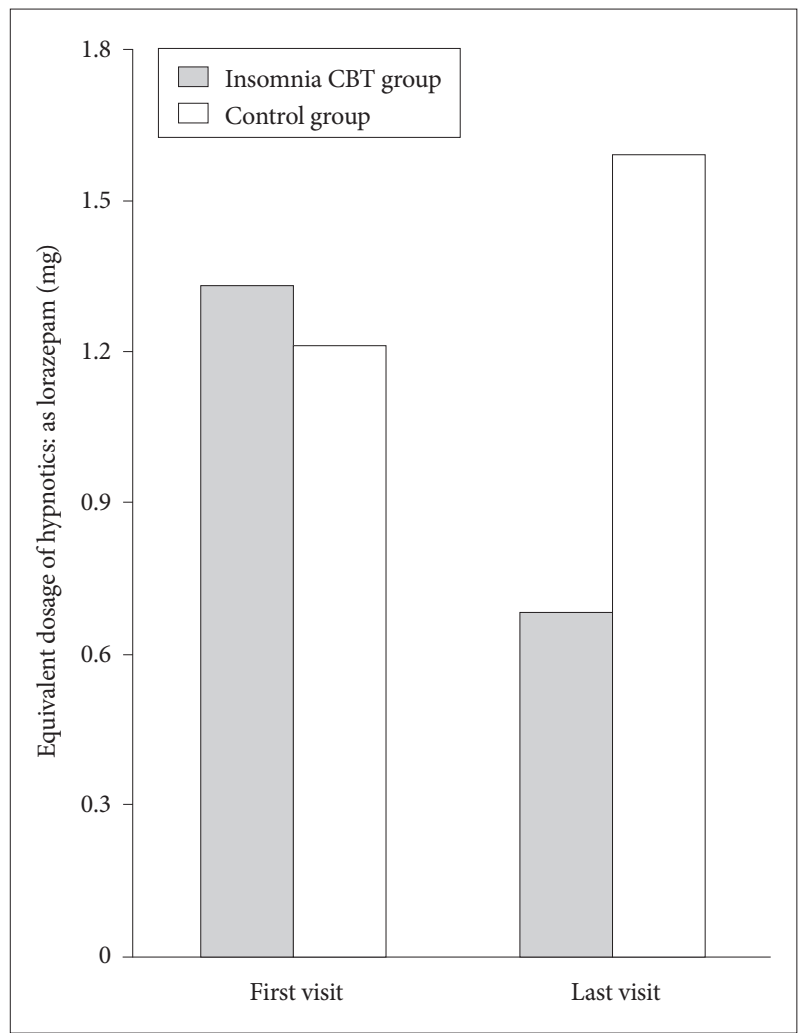

Figure 1. Prescription of hypnotics for insomnia. CBT: cognitive behavioral therapy.

At the first visit, no difference was found. However, at the last visit, the prescription rates of hypnotics and antidepressant in the CBT-i group were significantly lower than in the controls. We also examined the prescription rate and dosage of mirtazapine and trazodone, and found no differences at either the first or the last visit.

We examined changes in the dosages of prescribed sleep medications between the first and last visits using repeated measures of ANOVA. We found a significant change in group by time interaction on hypnotic dosage (Table 3). The dosage of hypnotics decreased over time in the CBT-i group; however, it remained the same in the control group (Figure 1). We
Table 4. Status of treatment at the last visit

\begin{tabular}{lrcc}
\hline & $\begin{array}{r}\text { CBT-i } \\
(\mathrm{N}=41)\end{array}$ & $\begin{array}{r}\text { Control } \\
(\mathrm{N}=100)\end{array}$ & $\mathrm{p}$ \\
\hline Case closure, N (\%) & $6(15)$ & $4(4)$ & \\
Lost to follow-up, N (\%) & $27(66)$ & $78(78)$ & 0.07 \\
Treatment maintenance, N (\%) & $8(20)$ & $18(18)$ & \\
\hline
\end{tabular}

CBT-i: cognitive behavioral therapy of insomnia

found no significant change in group by time interaction on the dosage of antidepressants over time.

\section{Treatment outcome}

We performed $\chi^{2}$ tests for CGI and status of case closure at the last visit for comparison of treatment outcomes. Achievement of case closure was better in the CBT-i group at the trend level (Table 4). However, the number of improved cases evaluated by CGI at the last visit was not significantly different between the two groups (80.6\% in the CBT-i group vs. $81 \%$ in the control group, $\mathrm{p}=0.17$ ).

\section{DISCUSSION}

We found CBT-i reduced the need for hypnotics among patients with insomnia. Previous studies show that CBT-i improves sleep efficiency, increases total sleep time, and reduces wake time after sleep onset. ${ }^{18}$ Thus, in contrast to most previous studies that focused on sleep characteristics, our study is unique because it examined the effect of CBT-i on the prescription of sleep medications routinely combined with CBT-i. ${ }^{27,28}$

Hypnotic use increases the risk of abuse, decreases cognitive function, and increases traffic accidents and falls in a dose-dependent manner. ${ }^{14,29}$ Thus, our finding that CBT-i decreased the prescription rate and dosage of hypnotics can help avoid those risks. Our result is consistent with that of a previous study showing a decrease in sleep medication use among patients receiving internet-based CBT-i..$^{30} \mathrm{Few}$ studies have examined the role of CBT-i in reducing the use of hypnotics. Belleville et al. ${ }^{31}$ reported outstanding benefits from CBT-i 
on tapering hypnotics in self-help insomniacs. On the other hand, Taylor et al. ${ }^{32}$ showed no change in hypnotic use after CBT-i among hypnotic dependent patients. We assume that the comorbid condition of hypnotic dependence made CBTi less effective. Our study is not comparable with Taylor's study because we excluded patients with substance abuse disorders. In another study showing no superior effect of CBT-i, total duration of follow-up was a relatively short 3 months. ${ }^{33}$ There are only five cases with reduced prescription dosage by more than half in their first month after CBT-i. However, during the follow-up period after CBT-i, which was 513 days in average, twelve out of 41 patients quit hypnotics. This is a consistent finding that the effect of CBT-i is incremental rather than immediate. ${ }^{16,17}$

Previous studies report that CBT-i is helpful for insomnia patients with major depressive disorder and is an effective augmentation therapy for depression itself. ${ }^{34}$ However, we found no significant change in antidepressant use after CBT-i, perhaps because of the low dosage of prescribed antidepressants in our study. Specifically, the prescribed antidepressant dosage was similar between groups and equivalent to a fluoxetine dose of 3.5-5 mg, which is lower than the therapeutic dosage for depressive disorder. Indeed, antidepressant dosages for primary insomnia tends to be lower than those for major depressive episodes. ${ }^{35}$ As only two patients in our study had comorbid major depressive disorder, we speculate that antidepressants were prescribed not for the depression per se, but rather for sleep disturbances. This may explain why we observed no change in antidepressant dosage in either group.

Previous studies showed that medical comorbidities such as cardiovascular disease, diabetes, cancer, and some medications can influence the insomnia state. ${ }^{36-39}$ Approximately 30\% of patients had medical comorbidities, which may be due to our hospital being a general hospital. We thought that these medical comorbid states may affect the results. However, we found medical comorbidity had no effect on the prescription rate and dosage of sleep medications in either group. Previous studies confirm the efficacy of CBT-i among insomnia patients with medical comorbidities. ${ }^{40,41}$ Even though some patients had cancer, there were no unplanned admissions. Therefore, we can speculate that CBT-i reduces hypnotic prescription in patients with medical comorbidities as well as in healthy insomniacs.

We speculate that the continued use of hypnotics after CBT$\mathrm{i}$ is associated with doctors' or patients' reluctance to stop medication because of the fear of insomnia recurrence. This fear may be due to the chronic nature of these sleep disturbances. In addition, the average follow-up duration of patients who received CBT-i was more than 500 days, which is also indicative of the chronic nature of their insomnia. Chronic insomnia may be maintained by its self-perpetuating nature, involving selective attention and monitoring, distorted perceptions of daytime deficits, and counterproductive safety behaviors. ${ }^{42} \mathrm{~A}$ previous study showed that the prognosis of insomnia patients after CBT-i was better when use of hypnotics was stopped entirely, than when using these drugs as needed. ${ }^{17}$ Although additional research is needed, our results indicate that doctors should try to stop prescribing hypnotics after CBT-i.

Our study has some limitations, mostly related to its retrospective design. First, our study has a small sample size. Because we included only patients who completed five sessions of CBT-i as confirmed by doctors' orders and payment statements, only 41 patients were included in the final cohort. Second, selection bias may have been present. In the clinic, doctors might have recommended CBT-i to patients with milder symptoms, greater motivation, or greater financial or time resources. However, there were no significant differences between groups in regard to clinical characteristics or medication dosages at the first visit, thus minimizing the possibility of selection bias. Last, we could not completely exclude cases with psychiatric or medical comorbidities because of our study being conducted in a general hospital setting. Although we found no effect of psychiatric or medical comorbidities on the results, there are still possibilities that either comorbidities or their treatments could affect the study results.

In summary, we show that CBT-i reduces the need for hypnotics in insomnia patients. This finding is important because CBT-i can reduce the risk of accidents and complications associated with hypnotic use. Our results indicate that CBT-i offers additional benefits beyond improving sleep characteristics, and thus provides further reasons to recommend CBT$i$ as a first-line treatment for insomnia.

\section{Acknowledgments}

This work was supported by the National Research Foundation of Korea Grant funded by the Korean Government (NRF-2017S1A3A2067165) and a grant of the Korean Mental Health Technology R\&D Project, Ministry of Health \& Welfare, Republic of Korea (HM15C0995).

\section{REFERENCES}

1. American Psychiatric Association. Diagnostic and Statistical Manual of Mental Disorders (DSM-V). Arlington, VA: American Psychiatric Association Publishing; 2013.

2. Cho HJ, Seeman TE, Kiefe CI, Lauderdale DS, Irwin MR. Sleep disturbance and longitudinal risk of inflammation: moderating influences of social integration and social isolation in the Coronary Artery Risk Development in Young Adults (CARDIA) study. Brain Behav Immun 2015; 46:319-326.

3. Kim TH, Carroll JE, An SK, Seeman TE, Namkoong K, Lee E. Associations between actigraphy-assessed sleep, inflammatory markers, and insulin resistance in the Midlife Development in the United States (MIDUS) study. Sleep Med 2016;27-28:72-79.

4. Lee E, Cho HJ, Olmstead R, Levin MJ, Oxman MN, Irwin MR. Persistent sleep disturbance: a risk factor for recurrent depression in com- 
munity-dwelling older adults. Sleep 2013;36:1685-1691.

5. Kallestad H, Hansen B, Langsrud K, Ruud T, Morken G, Stiles TC, et al. Impact of sleep disturbance on patients in treatment for mental disorders. BMC Psychiatry 2012;12:179.

6. Tobaldini E, Costantino G, Solbiati M, Cogliati C, Kara T, Nobili L, et al. Sleep, sleep deprivation, autonomic nervous system and cardiovascular diseases. Neurosci Biobehav Rev 2017;74:321-329.

7. Morin CM, LeBlanc M, Belanger L, Ivers H, Merette C, Savard J. Prevalence of insomnia and its treatment in Canada. Can J Psychiatry 2011; 56:540-548.

8. Roth T, Coulouvrat C, Hajak G, Lakoma MD, Sampson NA, Shahly V, et al. Prevalence and perceived health associated with insomnia based on DSM-IV-TR; International Statistical Classification of Diseases and Related Health Problems, Tenth Revision; and Research Diagnostic Criteria/International Classification of Sleep Disorders, Second Edition criteria: results from the America Insomnia Survey. Biol Psychiatry 2011;69:592-600.

9. Ohayon MM. Epidemiology of insomnia: what we know and what we still need to learn. Sleep Med Rev 2002;6:97-111.

10. Sadock BJ, Sadock VA, Ruiz P. Kaplan and Sadock's Synopsis of Psychiatry. Behavioral Sciences/Clinical Psychiatry Eleventh Edition. Philadelphia, PA: Lippincott Williams \& Wilkins; 2014.

11. Qaseem A, Kansagara D, Forciea MA, Cooke M, Denberg TD. Management of chronic insomnia disorder in adults: a clinical practice guideline from the American college of physicians. Ann Intern Med 2016;165:125-133.

12. Schutte-Rodin S, Broch L, Buysse D, Dorsey C, Sateia M. Clinical guideline for the evaluation and management of chronic insomnia in adults. J Clin Sleep Med 2008;4:487-504.

13. Olfson M, King M, Schoenbaum M. Benzodiazepine use in the United States. JAMA Psychiatry 2015;72:136-142.

14. Smink BE, Egberts AC, Lusthof KJ, Uges DR, de Gier JJ. The relationship between benzodiazepine use and traffic accidents: a systematic literature review. CNS Drugs 2010;24:639-653.

15. Wagner AK, Zhang F, Soumerai SB, Walker AM, Gurwitz JH, Glynn $\mathrm{RJ}$, et al. Benzodiazepine use and hip fractures in the elderly: who is at greatest risk? Arch Intern Med 2004;164:1567-1572.

16. Morin CM, Colecchi C, Stone J, Sood R, Brink D. Behavioral and pharmacological therapies for late-life insomnia: a randomized controlled trial. JAMA 1999;281:991-999.

17. Morin CM, Vallieres A, Guay B, Ivers H, Savard J, Merette C, et al. Cognitive behavioral therapy, singly and combined with medication, for persistent insomnia: a randomized controlled trial. JAMA 2009;301: 2005-2015.

18. Irwin MR, Cole JC, Nicassio PM. Comparative meta-analysis of behavioral interventions for insomnia and their efficacy in middle-aged adults and in older adults 55+ years of age. Health Psychol 2006;25:3-14.

19. Morin CM, Colecchi C, Stone J, Sood R, Brink D. Behavioral and pharmacological therapies for late-life insomnia: a randomized controlled trial. JAMA 1999;281:991-999.

20. Edinger JD, Colleen EC. Overcoming Insomnia: A Cognitive-Behavioral Therapy Approach, Therapist Guide. Second Edition. New York, NY: Oxford University Press; 2 edition; 2014

21. Morin CM, Vallières A, Guay B, Ivers H, Savard J, Mérette C, et al. Cognitive behavioral therapy, singly and combined with medication, for persistent insomnia: a randomized controlled trial. JAMA 2009;301: 2005-2015.

22. Ray WA, Griffin MR, Downey W. Benzodiazepines of long and short elimination half-life and the risk of hip fracture. JAMA 1989;262:3303-
3307.

23. Wiegand MH. Antidepressants for the treatment of insomnia: a suitable approach? Drugs 2008;68:2411-2417.

24. Hayasaka Y, Purgato M, Magni LR, Ogawa Y, Takeshima N, Cipriani A, et al. Dose equivalents of antidepressants: evidence-based recommendations from randomized controlled trials. J Affect Disord 2015; 180:179-184.

25. Wagstaff AJ, Ormrod D, Spencer CM. Tianeptine: a review of its use in depressive disorders. CNS drugs 2001;15:231-259.

26. Inada T, Inagaki A. Psychotropic dose equivalence in Japan. Psychiatry Clin Neurosci 2015;69:440-447.

27. Kaldo V, Jernelöv S, Blom K, Ljótsson B, Brodin M, Jörgensen M, et al. Guided internet cognitive behavioral therapy for insomnia compared to a control treatment - A randomized trial. Behav Res Ther 2015;71: 90-100.

28. Mitchell MD, Gehrman P, Perlis M, Umscheid CA. Comparative effectiveness of cognitive behavioral therapy for insomnia: a systematic review. BMC Fam Pract 2012;13:40.

29. Herings RM, Stricker BH, de Boer A, Bakker A, Sturmans F. Benzodiazepines and the risk of falling leading to femur fractures. Dosage more important than elimination half-life. Arch Intern Med 1995;155: 1801-1807.

30. Blom K, Jernelöv S, Rück C, Lindefors N, Kaldo V. Three-year followup of insomnia and hypnotics after controlled internet treatment for insomnia. SLEEP 2016;39:1267-1274.

31. Belleville G, Guay C, Guay B, Morin CM. Hypnotic taper with or without self-help treatment of insomnia: a randomized clinical trial. J Consult Clin Psychol 2007;75:325-335.

32. Taylor HL, Rybarczyk BD, Nay W, Leszczyszyn D. Effectiveness of a CBT intervention for persistent insomnia and hypnotic dependency in an outpatient psychiatry clinic. J Clin Psychol 2015;71:666-683.

33. O'Connor K, Marchand A, Brousseau L, Aardema F, Mainguy N, Landry P, et al. Cognitive-behavioural, pharmacological and psychosocial predictors of outcome during tapered discontinuation of benzodiazepine. Clin Psychol Psychother 2008;15:1-14.

34. Charles M, Morin M, Benca R. Chronic insomnia. Lancet 2012;379: 1129-1141.

35. Becker PM. Pharmacologic and nonpharmacologic treatments of insomnia. Neurol Clin 2005;23:1149-1163.

36. Kasasbeh E, Chi DS, Krishnaswamy G. Inflammatory aspects of sleep apnea and their cardiovascular consequences. South Med J 2006;99:5867.

37. Knutson KL, Ryden AM, Mander BA, Van Cauter E. Role of sleep duration and quality in the risk and severity of type 2 diabetes mellitus. Arch Intern Med 2006;166:1768-1774.

38. Davidson JR, MacLean AW, Brundage MD, Schulze K. Sleep disturbance in cancer patients. Soc Sci Med 2002;54:1309-1321.

39. Foral P, Knezevich J, Dewan N, Malesker M. Medication-induced sleep disturbances. Consult Pharm 2011;26:414-425.

40. Vitiello MV, Rybarczyk B, Von Korff M, Stepanski EJ. Cognitive behavioral therapy for insomnia improves sleep and decreases pain in older adults with co-morbid insomnia and osteoarthritis. J Clin Sleep Med 2009;5:355-362.

41. Johnson JA, Rash JA, Campbell TS, Savard J, Gehrman PR, Perlis M, et al. A systematic review and meta-analysis of randomized controlled trials of cognitive behavior therapy for insomnia (CBT-I) in cancer survivors. Sleep Med Rev 2016;27:20-28.

42. Harvey AG. A cognitive model of insomnia. Behav Res Ther 2002;40: 869-893. 